\title{
Serum concentrations of the axonal injury marker neurofilament light protein are not influenced by blood- brain barrier permeability
}

Authors: Marie Kalm ${ }^{a}$, Martina Boström ${ }^{b *}$, Åsa Sandelius ${ }^{c}$, Yohanna Eriksson $^{a}$, C. Joakim Ek ${ }^{d}$, Kaj Blennow ${ }^{c, e}$, Thomas Björk-Eriksson ${ }^{b}$ and Henrik Zetterberg, ${ }^{\text {,ce,f }}$

*equal contribution

${ }^{a}$ Department of Pharmacology, Institute of Neuroscience and Physiology, ${ }^{b}$ Department of Oncology, Institute of Clinical Sciences, ${ }^{\mathrm{C}}$ Department of Psychiatry and Neurochemistry, Institute of Neuroscience and Physiology, ${ }^{\mathrm{d} D e p a r t m e n t}$ of Physiology, Institute of Neuroscience and Physiology, Sahlgrenska Academy at the University of Gothenburg, Gothenburg, Sweden, ${ }^{\mathrm{e}}$ Clinical Neurochemistry Laboratory, Sahlgrenska University Hospital, Mölndal, Sweden, and the ${ }^{\mathrm{f}}$ Department of Molecular Neuroscience, UCL Institute of Neurology, Queen Square, London, United Kingdom

Corresponding author:

Marie Kalm, PhD, The Sahlgrenska Academy at the University of Gothenburg, Institute of Neuroscience and Physiology, Department of Pharmacology, S-405 30 Gothenburg, Sweden. Tel: (+46) 31-786 3425. E-mail: marie.kalm@ neuro.gu.se

\section{Highlights}

- There is an acute increase of serum NFL following both cranial irradiation and anesthesia in mice.

- The levels of serum NFL do not correlate with the acute opening of the BBB in a mouse model of cranial irradiation.

- Human data show that the levels of NFL in blood mirror the levels in CSF independently of the degree of BBB integrity. 


\begin{abstract}
A blood biomarker to monitor individual susceptibility to neuronal injury from cranial radiotherapy could potentially help to individualize treatment plans using radiation and thereby reduce the incidence and severity of late effects. An important aspect of such a blood biomarker is that its concentration is not confounded by varying degrees of release of the marker from the brain into the blood across the blood-brain barrier (BBB). In this study, we investigated serum neurofilament light (NFL) concentrations in 21-day old mice following a single dose of cranial irradiation ( $8 \mathrm{~Gy}$ ). Cranial irradiation resulted in acute cell injury measured as a 12.9-fold increase in caspase activity 6 hours after irradiation, inflammation, measured as XXX and increased BBB permeability, measured as XXX. Serum levels of NFL peaked at 6 hours after both anesthesia and cranial irradiation but there was no correlation of serum NFL concentration with BBB permeability over time. Further, three groups of patients with different degrees of BBB impairment (measured as the CSF/serum albumin ratio) were investigated. There was no correlation of serum NFL concentration with $\mathrm{CSF} /$ serum albumin ratio $(\mathrm{r}=\mathrm{X}, \mathrm{p}=\mathrm{Y})$ but a strong correlation of serum NFL concentration with NFL concentration in CSF ( $r=X, p=Y)$. In conclusion, serum NFL appears to be a reliable blood biomarker for neuronal injury, which is not influenced by BBB permeability.
\end{abstract}

\title{
Keywords
}

Biomarker, late effects, cranial radiotherapy, neurotoxicity juvenile brain

\section{Abbreviations}

CNS; central nervous system, CSF; cerebrospinal fluid, NFL; neurofilament light protein, CIR; cranial irradiation, BBB; blood-brain barrier, BCB; blood-CSF barrier, CCL2; chemokine (C-C motif) ligand 2, ICAM-1; intercellular adhesion molecule 1, E-selectin; Endothelial-selectin 


\section{Introduction}

The need to find reliable acute biomarkers in blood that reflects neuronal injury is of high interest in the clinical setting. To be able to make an early and accurate diagnosis it is important to know how the biomarker behaves and what factors that could influence the levels of brain specific biomarker, such as the function of the blood brain barrier (BBB). One group of patients that would benefit greatly from an acute biomarker to determine individual susceptibility to central nervous system (CNS) injury is pediatric patients with tumors located in the CNS. The most common forms of solid tumors in children are located in the CNS, with an annual incidence of 4.2/100,000 in children in Sweden (Lannering et al., 2009), and similar numbers in the U.S. (Ostrom et al., 2014). Tumor histology highly influences the survival rates; for example the five-year survival rate is 94.1\% for pilocytic astrocytoma but only 5.0\% for glioblastoma (Ostrom et al., 2014). However, independent on the difference in survival rates, survivors are often living with severe and debilitating consequences of the treatment that they received when they were children, the socalled "late effects". For example, treatment of brain tumors significantly decreases the likelihood of being employed, getting married or having close friends (Gurney et al., 2009). Although all the treatment modalities (surgery, chemotherapy, and radiotherapy) cause negative complications, radiotherapy has the highest severity of late effects (Han et al., 2009). Cognitive impairment is one of the most severe complications after cranial radiotherapy for both children and adults (Duffner, 2004; Duffner, 2010; Makale et al., 2017; Mulhern et al., 2004). Radiotherapy, the volume of brain tissue treated, young age at diagnosis and female gender are some risk factors for late effects (Fouladi et al., 2005; Han et al., 2009; Lahteenmaki et al., 2007). The precautions taken are not enough to prevent late effects and as of today, there is no way to determine individual susceptibility to cranial radiotherapy. The primary goal of modern treatment strategies is to improve the chance of survival and secondly improve the patients' future quality of life. With the increasing number of survivors as a result of improved treatment strategies, it is important to also consider their future health and individual susceptibility when designing the treatment plan.

An acute biomarker to determine individual susceptibility to CNS injury from cranial radiotherapy could potentially help to individualize treatment plans and reduce the incidence of late effects. Our results from preclinical irradiation models have demonstrated that inflammatory mediators such as the chemokine (C-C motif) ligand 2 (CCL2) could be correlated with the injuries seen on stem 
cells in the hippocampus after cranial irradiation (CIR) to mice (Roughton et al., 2013). In the clinical setting, we have seen that the neuronal injury marker neurofilament light protein (NFL) increased by $120 \%$ in cerebrospinal fluid (CSF) three months after prophylactic radiotherapy to the brain of small cell lung cancer patients (Kalm et al., 2014). However, since lumbar puncture often necessitates anesthesia for children, blood-based analyses are a more suitable and useful alternative for clinical monitoring. We have recently developed an ultra-sensitive assay for NFL in plasma and serum (Gisslen et al., 2016). Using this assay we have shown that plasma/serum NFL concentrations increased in HIV-associated dementia (Gisslen et al., 2016), frontotemporal dementia (Rohrer et al., 2016) and Alzheimer's disease (Mattsson et al., 2017), and correlate with longitudinal changes in brain volumes and cognitive functioning (Rohrer et al., 2016). Further, serum NFL concentration increases acutely and stays elevated for months following both severe and mild traumatic brain injury with correlations to both injury severity, as well as imaging measures of neuroaxonal injury and outcome (Ljungqvist et al., 2016; Shahim et al., 2016). Due to all of these previous studies, we consider NFL as a promising blood biomarker for neuronal injury following CIR. An important aspect of such a biomarker, however, is that its concentration is not confounded by blood-brain barrier (BBB) permeability.

In the current study, we examine the correlation of serum NFL concentrations with markers of neuronal injury, inflammation and BBB integrity in mice following CIR. We also assess the potential influence of BBB permeability on serum NFL concentrations in patients with varying degrees of BBB impairment.

\section{Results}

1.1 CIR-induced injury response and cross-talk between the BBB and the immune system in mice

To evaluate CIR-induced injury, we assessed a cell death parameter, inflammation markers and BBB permeability. First, cell death was investigated by a caspase activity assay (DevDase). We observed a 12.9-fold increase of caspase activity in brain tissue, indicative of apoptosis, 6 hours after CIR compared to untreated control mice (Fig. 1A, P<0.001). Further, at the 12-hour timepoint after CIR, there was still a significant increase of caspase activity compared to the control 
levels $(\mathrm{P}=0.005)$. However, at this time-point, the increase was only 2.7 -fold and at the 24-hour time-point, caspase activity had normalized.

The inflammatory response was measured by CCL 2 expression and was significantly increased by $130 \%$ at 6 hours after CIR compared to control mice (Fig. 1B, P<0.001). Compared to the cell death analysis, the levels of CCL2 continued to increase and reached the peak at 12-hour and 24hour post CIR, when the levels of CCL2 had increased with 194\% and 190\%, respectively, compared to the control group $(\mathrm{P}<0.001)$. Not until 48 hours after CIR did the levels drop down to $50 \%$ above the controls $(\mathrm{P}=0.002)$, and one day further the levels were no longer different from the controls (Fig. 1B).

\section{Figure 1}
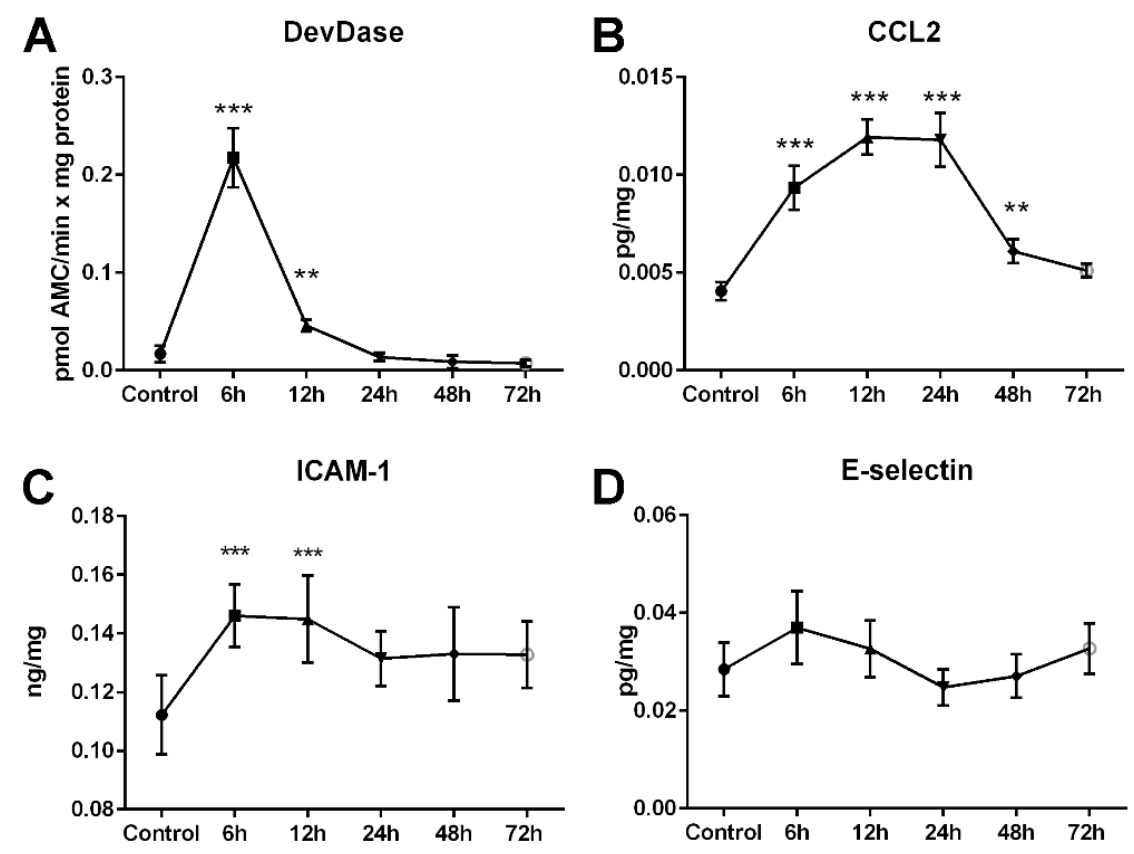

Figure 1. The levels of caspase 3/7 activity (A), CCL2 (B), ICAM-1 (C) and E-selectin (D) were measured in brain homogenates at different time-points after CIR to the young mouse brain (P21). Data shown as mean \pm SEM. $N=8$ Controls, $6_{C I R .} P<0.01 * *, P<0.001^{* * *}$. Only statistical differences between the control and the CIR groups are shown in the histogram.

We also investigated classical markers for BBB and immune system cross-talk after CIR (Fig. 1CD). Intercellular adhesion molecule 1 (ICAM-1) expression was increased with $30.1 \%$ at the first 
time-point, 6 hours, compared to the control group (Fig. 1C, P<0.001). This increase stayed more or less unchanged at the 12-hour time-point $(29.1 \%, \mathrm{P}<0.001)$. 24 hours after CIR the levels were only $17 \%$ higher than the controls (n.s. $\mathrm{P}=0.14$ ). The levels of ICAM-1 however stayed on that level for the duration of the experiment (48-hours post-CIR, n.s. $\mathrm{P}=0.077$ and 72-hours post-CIR, n.s. $\mathrm{p}=0.085$ ). E-selectin, a protein involved in the rolling of leukocytes along the endothelial, was increased by $29.9 \%$ at 6 hours after CIR, however this was not significant compared to controls due to a high standard error within the groups (Fig. 1D). The data however indicate that there is a recruitment of immune cells to the brain acutely after CIR since the 6-hour time-point had 49.3\% higher E-selectin expression compared to the 24-hour time-point ( $\mathrm{P}=0.008)$.

\subsection{CIR-induced BBB permeability in mice}

We measured ${ }^{14} \mathrm{C}$-sucrose brain and CSF uptake ratios to assess blood-CSF and BBB permeability after CIR with ratios corrected for residual blood volume. Radiation resulted in an opening of the BBB with 103\% at 24 hours after CIR compared to controls in the entire brain (Fig. 2A, P<0.001). The accumulation of ${ }^{14} \mathrm{C}$-sucrose was increased by $103 \%$ in the cortex (Fig. 2B, $\mathrm{P}<0.001$ ), $108 \%$ in the striatum/thalamus (Fig. $2 \mathrm{C}, \mathrm{P}=0.0013$ ), $87.8 \%$ in the brain stem (Fig. $2 \mathrm{D}, \mathrm{P}<0.001$ ), $106 \%$ in the hippocampus (Fig. 2E, $\mathrm{P}=0.001$ ), and $105 \%$ the cerebellum (Fig. $2 \mathrm{~F}, \mathrm{P}<0.001$ ). In addition, the levels of ${ }^{14} \mathrm{C}$-sucrose in the CSF showed an increase of $39.1 \%$ at the 24-hour time-point compared to the control levels (Fig. 2G, $\mathrm{P}=0.0090$ ). There was a similar opening in all measured brain regions with an indication that the opening started around 12 hours (however not significant at this time-point) and reached a permeability peak at 24 hours after CIR. At the 48-and 72-hour time-points, the permeability of the BBB was returned to control levels. 
Figure 2
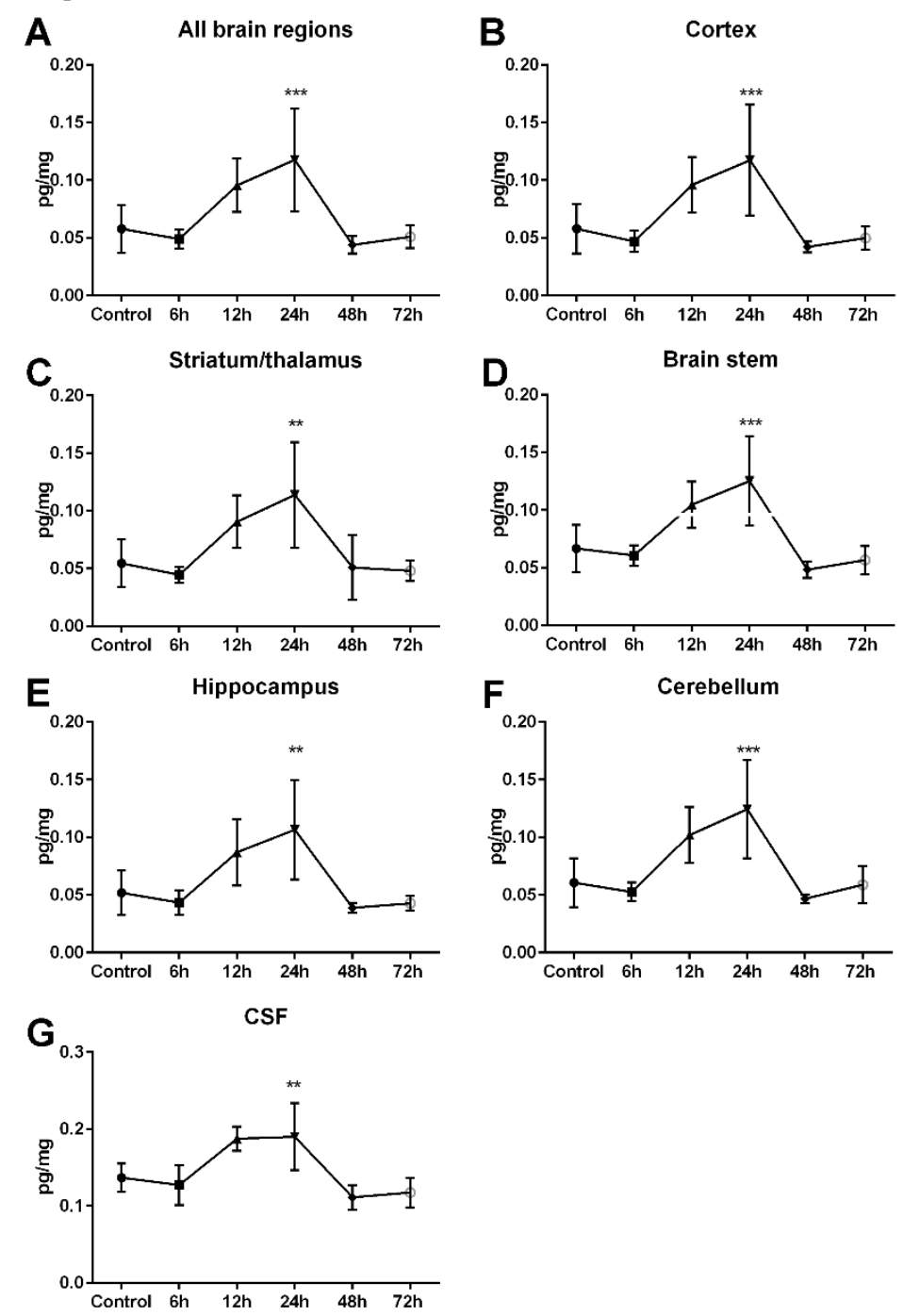

Figure 2. The uptake of ${ }^{14} C$-sucrose over the BBB was measured at different time-points after CIR to the young mouse brain (P21). The concentration of ${ }^{14} C$-sucrose is presented in the entire brain $(A)$, cortex $(B)$, striatum/thalamus $(C)$, brain stem $(D)$, hippocampus $(E)$, cerebellum $(F)$ and in the $\operatorname{CSF}(G)$. Data shown as mean \pm SEM. N=10 Controls, 5-7 CIR. $P<0.01^{* *}, P<0.001^{* * *}$. Only statistical differences between the control and the CIR groups are shown in the histogram. 


\subsection{Neurofilament light protein serum concentration and correlation with blood-brain barrier}

permeability in mice

Blood samples were collected from the mice pups during the acute phase following CIR (Fig. 3). Serum NFL concentrations were the highest at the first collection time point. Although a timedependent decline in NFL concentration following CIR was observed, the same trend was seen also in the control group that had only been sedated with tribromoethanol and not subjected to CIR. To investigate if the anesthetic drug influenced serum NFL levels, naïve control groups were consequently added to the experiment. These mice were brought to the irradiation facility at the hospital but were not subjected to anesthesia or irradiation. The results showed that only the sedated and CIR groups had higher concentrations of NFL in the blood, whilst naïve controls had unaltered low concentrations throughout the experiment (Fig. 3). Furthermore, only the CIR treated groups were significantly different over time compared to the naïve controls $(\mathrm{P}=0.003)$. At the 6-hour time-point the CIR group had a mean of $55.9 \mathrm{pg} / \mathrm{ml}$ whereas the anesthetized controls had a mean of $39.4 \mathrm{pg} / \mathrm{ml}$ and the naïve control mice a mean of $10.5 \mathrm{pg} / \mathrm{ml}$. Importantly, NFL concentrations in blood did not correlate with the timing of BBB opening (Fig. 2).

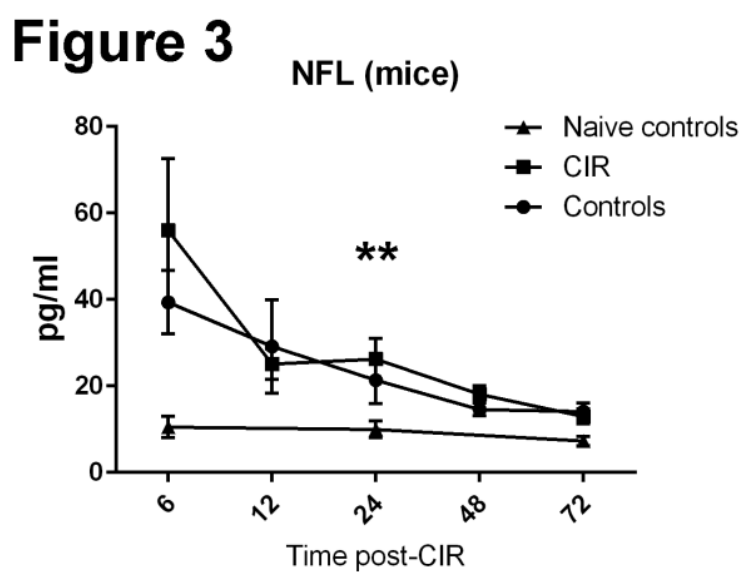

Figure 3. The levels of serum NFL were measured in mice at different time-points after CIR to the young mouse brain (P21). Data shown as mean $\pm S E M$. N=6-11 per group. ** $P<0.01$, comparison between CIR and nä̈ve controls over time. 


\subsection{Neurofilament light protein serum concentration and correlation with blood-}

brain barrier permeability in humans

To examine the relationship between serum NFL concentration and BBB permeability further, we measured CSF/serum albumin ratio (a BBB marker) and serum and CSF NFL concentrations in humans with varying degree of BBB impairment (Supplementary table 1, Fig. 4A-B). The groups were divided into those with an albumin ratio below 10 (normal range), a midrange group with a ratio of 10-20 and a high group with a ratio above 20 (severe BBB impairment). Although individuals with high CSF/serum albumin ratio often had higher concentrations of both CSF (Fig 4A) and serum NFL (Figure 4B) concentrations compared with individuals with normal $\mathrm{CSF} /$ serum albumin ratio), there was no correlation of serum NFL concentration with CSF/serum albumin ratio (Figure 4C). In contrast, serum NFL concentrations correlated tightly with NFL concentrations in the CSF (Fig. 4D).

\section{Figure 4}
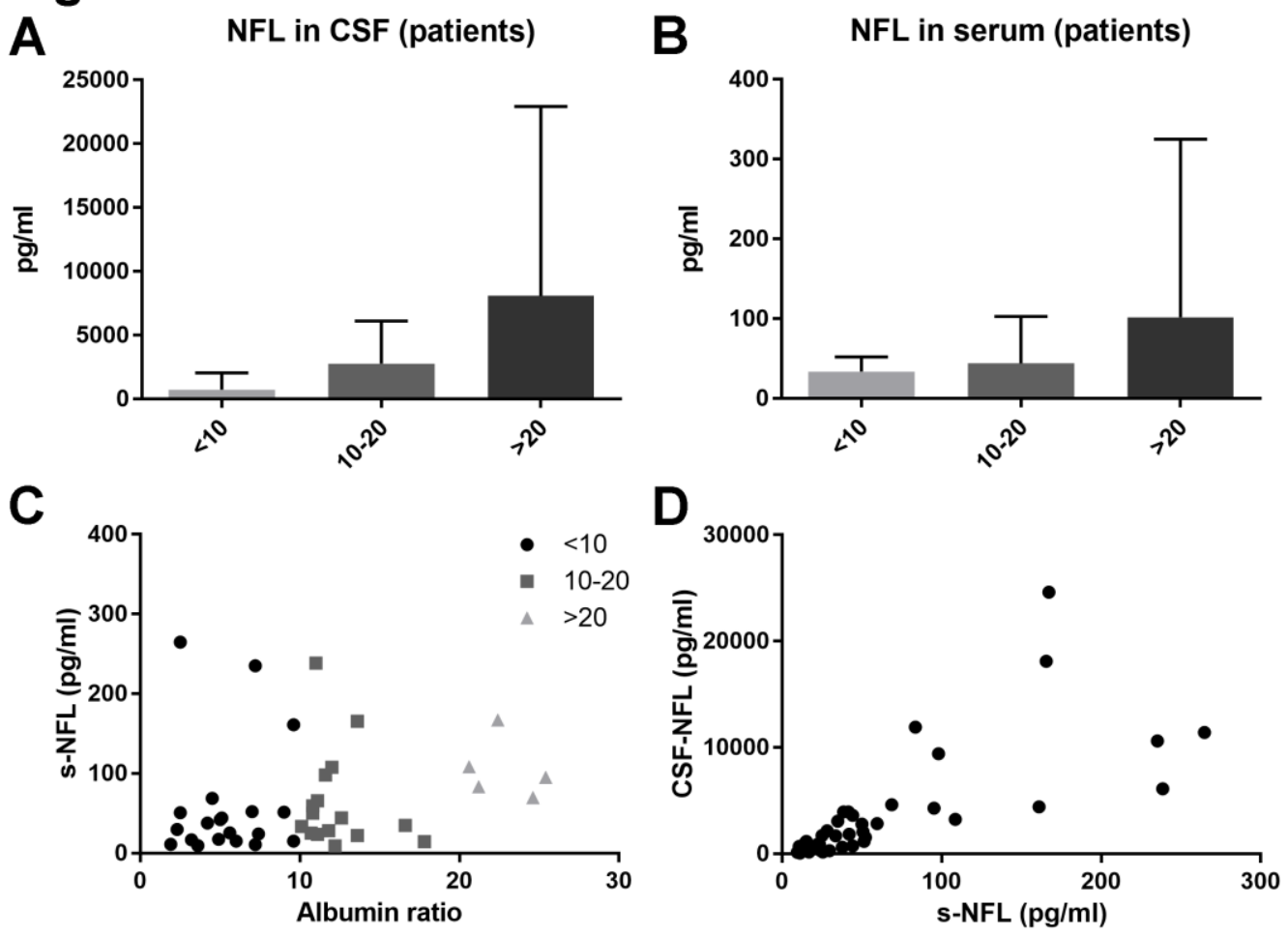
Figure 4. The levels of NFL were measured in CSF (A) and serum (B) from humans with different blood-brain barrier permeability. A CSF/serum albumin ratio $<10$ represents normal blood-brain barrier integrity $(n=X), 10-20$ medium permeability $(n=X),>20$ has a high permeability $(n=X)$. Data is presented as median \pm interquartile range $(C)$. Patients with a CSF/serum albumin ratio lower than 30 and serum NFL lower than 400pg/ml are presented (six data points are missing, but presented in the supplementary table 1). No difference was seen between the groups and the level of serum NFL. (D) The levels of NFL in serum did reflect the NFL concentrations in CSF across groups with different degrees of blood-brain barrier permeability ( $r=0.6303, p<0.0001$; all samples). To visualize the correlation only concentrations lower that $30.000 \mathrm{pg} / \mathrm{ml}$ in CSF and lower than $300 \mathrm{pg} / \mathrm{ml}$ are shown in the scatter plot (three data points are missing but presented in the supplementary table 1).

\section{Discussion}

This study aimed to investigate if it was possible to use serum NFL concentration as a biomarker for neuronal injury following CIR. The major findings were as follows: 1) there is an acute increase in serum NFL concentration in mice following both CIR and anesthesia, 2) serum levels of NFL in mice did not correlate with the opening of the BBB following CIR, 3) NFL concentration in serum reflects CSF NFL concentration independently of BBB permeability in patients with varying degrees of BBB impairment. Although the reasons for the increase in serum NFL after anesthesia needs further examination, these data suggest that the marker reflects CSF NFL concentration without being confounded by BBB permeability.

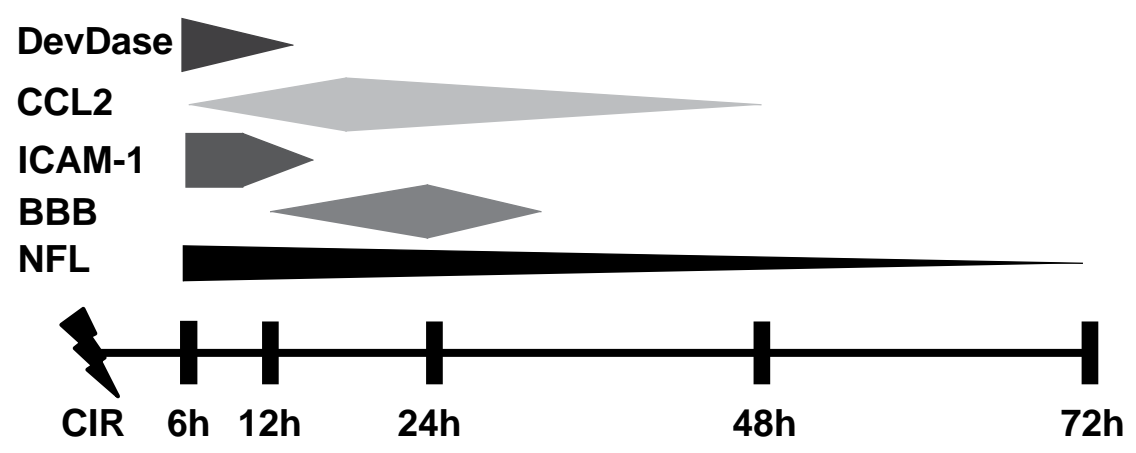


Figure 5. Schematic overview of the process of CIR-induced injury response over time.

The mice used in the current study were 3 weeks old (P21) and correlate developmentally with a 3 year old child (Semple et al., 2013). Due to this, the brains are highly sensitive to CIR, which can be visualized in the cell death measurement that peaked at 6 hours post-CIR. This can be compared to the adult rodent brain where cell death was not measurable at this time after CIR (Blomstrand et al., 2014). Furthermore, we have previously shown that CIR induces a transient inflammatory response (Kalm et al., 2009). Chemokines are small peptides that are secreted during neuronal injury and play an important role in migration, leukocyte trafficking, and intracellular communication during neuroinflammation (Tambuyzer et al., 2009; Ubogu et al., 2006). CCL2 is the chemokine that shows the highest upregulation following CIR to the developing brain (Kalm et al., 2009). In this study, CCL2 showed a rapid upregulation and stayed elevated for 48 hours, hence 24 hours after the cell death levels had returned back to normal. Interestingly, the levels did not decrease until the integrity of the BBB had been restored. A large number of molecules could affect the permeability of the BBB and in this study the timeline correlates with the upregulation of both CCL2 and ICAM-1 (Overview, Fig. 5). CCL2 has been shown to disrupt the integrity of the BBB, and could therefore play an important role in disease progression (reviewed in Yao and Tsirka, 2014). Another step in the injury process following CIR, which could be of importance for the permeability of the BBB, is the recruitment of neutrophils. The level of ICAM-1 increased at the first time-point after CIR and stayed elevated during the duration of the experiment. ICAM-1 is a key factor in trafficking neutrophils to sites of injury (Lyck and Enzmann, 2015). Slowing the neutrophils down in the blood circulation is a multistep procedure but is mediated by selectins on the endothelial surface (Zarbock et al., 2011). In this study, E-selectin followed the same expression pattern as ICAM-1, but there were no significant changes compared to baseline. There was however a significant difference between the 6-hour time-point and 24-hour time-point after CIR. The process of recruiting immune cells to the brain following CIR presumably affects the increased BBB permeability observed in the current study.

In this study, a sensitive method with ${ }^{14} \mathrm{C}$-sucrose was used to investigate the BBB permeability after CIR to the developing mouse brain. It has been shown that irradiation of the brain increases permeability of cerebral vessels (evaluated by Evans blue staining) at 1 and 4 weeks after injury 
in both young and adult mice (Sandor et al., 2014). Interestingly, Sandor et al. did investigate the permeability one day following CIR but could not detect any increased permeability. The difference between their results and the results observed in the current study could be due to the fact that Evans blue (EB) is a less sensitive method to measurement of BBB permeability compared to ${ }^{14} \mathrm{C}$-sucrose. Another possible explanation to this discrepancy is that EB binds to plasma proteins in blood and thus sucrose (342Da) is a much smaller molecule than the EB-plasma protein complexes. In this study, we wanted to investigate the acute phase following CIR to be able to correlate cell death, inflammation, BBB permeability and the optimal time-window to detect NFL in blood. Our data show that the BBB is affected 24 hours after a single dose of CIR to the young (P21) mouse brain. This knowledge could be of great importance, for example when combining drug therapies, such as chemotherapy, with radiotherapy toward the brain. Our data show that the $\mathrm{BBB}$ is opened with a time lag after CIR, which is of importance in the context of administering drugs simultaneously as CIR treatment. Since radiotherapy is given on weekdays (paused on weekends due to practical reasons), the effects of drug administered the first day of a week would in this aspect not be comparable if it instead was given the last day. Consequently, the amount of drug needed to reach the wanted concentration and effect in the brain the last day would presumably be lower compared to if the drug was given on the first day of treatment. Nevertheless, the BBB is highly affected by CIR and this could be explained by the injury and immune response.

Neurofilaments are the major component of axonal cytoskeleton proteins, important for both morphologic integrity and conduction of nerve pulses along axons of neurons (Lee and Cleveland, 1996). They consist of three subunits that differ in size where NFL is the smallest subunit. NFL is a well-examined biomarker in CSF for injury of large-caliber myelinated axons projecting into deeper brain layers and the spinal cord (Friede and Samorajski, 1970; Lee and Cleveland, 1996; Petzold, 2005). To monitor and predict late effects after CIR, a fluid biomarker that can reflect the injury process is of crucial importance. We have seen that NFL is highly elevated in CSF after prophylactic cranial radiotherapy in patients (Kalm et al., 2014). In addition, in this study we show that the levels of NFL in human CSF and blood correlate very well, without any influence by the integrity of the BBB. The NFL measurement in blood did peak at the 6-hour time-point in our mouse model of CIR and decreased successively afterwards. Another finding of importance was that anesthesia with tribromoethanol also showed a similar elevation of NFL in blood. Cell death 
was however not detected in that group and could thereby not explain the results. By adding a naïve control group, we could exclude that stress was influencing the levels of NFL. This knowledge is hence important to consider when designing a mouse study and when selecting anesthesia. Nevertheless, tribromoethanol has an effect on neuronal integrity or function that can be measured in blood from mice. In a clinical study, peripheral injury (surgery and anesthesia) did not change the levels of NFL in CSF (Anckarsater et al., 2014), supporting that the effects of tribromoethanol presumably are mild. To continue, our findings suggest that NFL is a sensitive marker for neuronal injury. Others have also described NFL as a reliable and accessible biomarker to monitor neurodegenerative disease in mouse models (Bacioglu et al., 2016). In patients, serum NFL has been described as a potential biomarker for other neurological diseases, such as multiple sclerosis (Kuhle et al., 2016b), amyotrophic lateral sclerosis (Weydt et al., 2016) and progressive supranuclear palsy (Rojas et al., 2016). The levels of NFL in CSF and blood do correlate very well. With that in mind, Gisslén et al. have been able to detect NFL in blood of patients with HIV infection and found a strong correlation with NFL levels in CSF as well as a correlation with the severity of CNS infection (Gisslen et al., 2016). Further studies are needed to investigate if NFL can be used to predict the late effects following cranial radiotherapy.

In conclusion, we examined how the BBB is affected during the acute time-window after CIR and if this affects the levels of serum NFL. CIR-induced cell death was followed by immune activation and increased permeability of the BBB. Despite our finding regarding tribromoethanol, the levels of NFL in serum did not correlate with a disturbed integrity of the BBB. These finding were also supported with human data showing that serum NFL was comparable to NFL in CSF, without any correlation with BBB permeability. Future studies are needed to verify how serum NFL is altered when CIR is given in a fractionating matter and under other anesthetic conditions. In summary, serum NFL has a good potential to act as a marker for neurotoxicity following cranial radiotherapy.

\section{Experimental procedures}

\subsection{Animals}

Female C57BL/6 mice from Charles River Laboratories, Sulzfeld, Germany, were used in this study. The animals were ordered in litters containing 6 pups per litter. All animals were kept on a 
12-hour light cycle with food and water provided ad libitum. All animal experiments were approved by the Gothenburg committee of the Swedish Animal Welfare Agency (2015-72).

\subsection{Cranial irradiation procedure}

A linear accelerator (Varian Clinac 1600 C-2 (250mu/min) and True Beam STX (600mu/min), Radiation Oncology Systems, LLC, San Diego, CA, USA) with 6 MV nominal photon energy was used for CIR. All animals were anesthetized on postnatal day (P) 21 with an intraperitoneal injection of $50 \mathrm{mg} / \mathrm{kg}$ of tribromoethanol (Sigma, Stockholm, Sweden) to immobilize the animals during the procedure. The animals were placed in a prone position on a polystyrene bed and the head was covered with a tissue equivalent material to obtain an even irradiation dose throughout the underlying tissue. Further, the whole brain was irradiated with $8 \mathrm{~Gy}$, using a radiation field of $2 \times 2 \mathrm{~cm}$ and a source to skin distance of $99.5 \mathrm{~cm}$. The variation of the absorbed dose was estimated to $\pm 5 \%$. An absorbed dose of $8 \mathrm{~Gy}$ is equivalent with $18 \mathrm{~Gy}$ delivered in 2-Gy fractions according to the linear quadratic formula and an alpha/beta ratio of 3 for late effects in the normal brain tissue (Fowler, 1989). The dose used for treatment of brain tumors in children, such as medulloblastoma, is higher, up to $55 \mathrm{~Gy}$ to the tumor bed. After the CIR procedure, the pups were placed on a warm bed $\left(\sim 36^{\circ} \mathrm{C}\right)$ to be able to maintain the body temperature of the animals. When the pups had recovered from the anesthesia, they were returned to their dams in their original cages until sacrificed. Control animals used in the experiments were anesthetized but not subjected to CIR. Naïve control animals were also used for the blood analysis of NFL. These mice were brought to the irradiation facility at the hospital but neither subjected to anesthesia nor CIR.

\subsection{Tissue preparation}

The brains were quickly removed after sacrifice, put in liquid nitrogen, and stored at $-80^{\circ} \mathrm{C}$. Brains were homogenized by sonication in phosphate-buffered saline (PBS) containing Triton X-100, ethylenediaminetetraacetic acid (EDTA, Sigma) and protease inhibitor cocktail (cOmplete, EDTA-free, ref 11873580001, Roche). After sonication, the samples were centrifuged and the cytosolic fractions were stored at $-80^{\circ} \mathrm{C}$. The total protein concentration was measured using a spectrophotometer (NanoDrop, ND-1000, Saveen Werner) before running the immunoassays. 


\subsection{Blood collection from mice}

Mice were anesthetized with a mixture of isoflurane and oxygen before blood collection. The chest was opened and blood was drawn from the heart with a $1 \mathrm{ml}$ syringe (Omnifix ${ }^{\circledR}$, BRAUN, Melsungen, Germany). Blood samples were immediately centrifuged for 5 minutes, serum was collected, transferred to a new tube and stored at $-80^{\circ} \mathrm{C}$.

\subsection{Fluorometric assay of caspase-3-like activity}

To measure CIR-induced caspase-dependent cell death, we used a caspase activity assay. Samples, $20 \mu \mathrm{l}$, were added to a microplate and mixed with $80 \mu \mathrm{l}$ extraction buffer (50 mM Tris-HCL, pH 7.3; $100 \mathrm{mM} \mathrm{NaCl} ; 5 \mathrm{mM}$ EDTA; $1 \mathrm{mM}$ EGTA; 0.2\% 3-[(3-cholamidoprophylene) dimethylamonio]propane sulphonic acid; $1 \%$ protease inhibitor cocktail (cOmplete, EDTA-free, ref 11873580001, Roche); 1 mм phenylmethylsulfonylfluoride). After incubation for 15 minutes at room temperature, $100 \mu \mathrm{l}$ peptide substrate, $25 \mu \mathrm{m}$ Ac-Asp-Glu-Val-Asp-aminomethyl coumarine (Ac-DEVD-AMC; (\#SAP3171v, Peptide Int.)) in assay buffer (50 mм Tris-HCl, pH 7.3; 100 тм NaCl; 5 тм EDTA; 1 mм EGTA; 1 mм phenylmethylsulfonylfluoride, $10 \mathrm{mM}$ dithiothreitol) was added to the samples. Cleavage of the substrate was measured at $37^{\circ} \mathrm{C}$ using Spectramax Gemini microplate fluorometer (Molecular Devices, Sunnyvale, CA) with an excitation wavelength of $380 \mathrm{~nm}$ and emission wavelength of $460 \mathrm{~nm}$. The degradation was followed at 2-minute intervals for 1 hour, and V-max was calculated from the entire linear part of the curve. Standard curves with AMC in the appropriate buffer were used to express the data in picomoles of AMC (7-amino-4-methyl-coumarin) formed per minute and per milligram of protein.

\subsection{Enzyme-linked immunosorbent assay}

Enzyme-linked immunosorbent assays (ELISA) were used to investigate chemokine (C-C motif) ligand 2 (CCL2, MJE00, R\&D Systems), Endothelial (E)-selectin (MES00, R\&D Systems), intercellular adhesion molecule 1 (ICAM-1, MIC100, R\&D Systems) expression after CIR. The analyses were performed according to the instructions of the manufacturers and the amount of the investigated proteins was measured using SpectraMax i3x (Molecular Devices, Sunnyvale, CA), with an excitation wavelength of $380 \mathrm{~nm}$ and emission wavelength of $460 \mathrm{~nm}$. 


\subsection{Blood-brain and blood-CSF barrier permeability}

Measurements of BBB and blood-CSF barrier (BCB) permeability was performed similar to our previous studies (Ek et al., 2015) at 6-hours ( $\mathrm{n}=5), 12$-hours ( $\mathrm{n}=5)$, 24-hours ( $\mathrm{n}=7), 48$-hours ( $\mathrm{n}=5$ ) and 72-hours $(\mathrm{n}=5)$ after CIR at P21 along with controls $(\mathrm{n}=10)$ which were mixed littermates from all time-points. ${ }^{14} \mathrm{C}$-sucrose (342Da; American Radiochemicals) was injected intraperitoneally $(0.2 \mu \mathrm{Ci} / \mathrm{g}$ body weight; $10 \mu \mathrm{l}$ injection/g animal) and animals killed at 27 minutes after the injections with an i.p. injection of pentobarbital. Blood was collected from the heart and plasma was separated by centrifugation. CSF was collected from cisterna magna (4-6 $\mu 1)$ as previously described (Ek et al., 2001). The brain was dissected out and the hippocampus, cortex, striatum/thalamus and brain stem were collected in pre-weighed scintillation vials. Brain samples were digested with SOLVABLETM (Perkin-Elmer). Scintillation fluid (Ultima-Gold, PerkinElmer) was added to the vials and the radioactivity in all samples determined by liquid scintillation counting. Tissue radioactive concentrations were corrected for residual blood volume space to obtain true tissue concentrations as previously described (Bolouri et al., 2014). Brain/blood and $\mathrm{CSF} /$ blood concentration ratios were calculated as an index of $\mathrm{BBB}$ and $\mathrm{BCB}$ permeability.

\subsection{Clinical samples}

Paired lumbar CSF and serum samples from clinical laboratory routine were selected to represent different degrees of BBB dysfunction. The selection was based on the CSF/serum albumin ratio, the best-established clinical biomarker for blood-brain barrier function (Tibbling et al., 1977). Following selection, the samples were terminally de-identified and analyzed for CSF and serum NFL as described below. Biochemical data are given in Supplementary table 1. The age and gender distribution was comparable between the groups. All procedures were approved by the Regional Ethics Committee at the University of Gothenburg.

\subsection{CSF/serum albumin ratio (humans)}

Albumin levels in serum and CSF were measured by immunonephelometry on a Beckman Immage Immunochemistry system (Beckman Instruments, Beckman Coulter, Brea, CA). The albumin ratio was calculated as CSF albumin (mg/l)/serum albumin ( $\mathrm{g} / \mathrm{l})$ and was used as a measure of the BBB function. 


\subsection{CSF NFL (humans)}

CSF NFL concentration was measured using the NF-Light ELISA, as described by the kit manufacturer (UmanDiagnostics, Umeå, Sweden).

\subsection{Serum NFL (mice and humans)}

The amino acid sequence of NFL is $100 \%$ conserved between rodents and humans. Serum sample NFL concentration was determined using an in-house NFL assay on the Single molecule array (Simoa) platform, which has been described in detail previously (Kuhle et al., 2016a). Briefly, paramagnetic carboxylated beads (Quanterix Corp, Boston, MA, USA) were coated with a mouse anti-neurofilament light antibody (UD1, UmanDiagnostics, Umeå, Sweden) and incubated 35 minutes with sample and a biotinylated mouse anti-neurofilament light antibody (UD2, UmanDiagnostics) in a Simoa HD-1 instrument (Quanterix). The bead-conjugated immunocomplex was thoroughly washed before incubation with streptavidin-conjugated $\beta$ galactosidase (Quanterix). After additional washes, resorufin $\beta$-D-galactopyranoside (Quanterix) was added and the immunocomplex was applied to a multiwell array designed to enable imaging of every single bead. The average number of enzymes per bead (AEB) of samples was interpolated onto the calibrator curve constructed by AEB measurements on bovine NFL (UmanDiagnostics) serially diluted in assay diluent. Samples were analyzed using one batch of reagents and animal treatment information was blinded to the one performing the analysis. The average repeatability of the assay was assessed by measurements of quality control samples and the coefficient of variation was $4.2 \%$ for a sample with a mean NFL concentration of $20.1 \mathrm{pg} / \mathrm{ml}$, and $6.2 \%$ for a sample with a mean NFL concentration of $50.0 \mathrm{pg} / \mathrm{ml}$. Similarly, the inter-assay coefficient of variation was $5.0 \%$ and $6.8 \%$ for each quality control sample. The limit of detection (LOD), determined as the mean blank signal +3 SD for the Simoa NFL assay was $0.3 \mathrm{pg} / \mathrm{ml}$ and the lower limit of quantification (LLOQ) determined as the mean blank signal + 10SD was $2.7 \mathrm{pg} / \mathrm{ml}$ when compensated for a four-fold sample dilution.

\subsection{Statistics}

For statistical analysis of DevDase, CCL2, ICAM-1, E-selectin and NFL (humans), one-way ANOVA with Bonferroni post hoc testing was used. NFL for the mice was analyzed with a two- 
way ANOVA with time and treatment as main effects. All analyses on mice data were performed using SPSS 23.0 (SPSS, Chicago, IL, USA) and statistical significance was considered if $\mathrm{P}<0.05$. The clinical correlation analysis between serum and CSF NFL was performed using Pearson $r$ (GraphPad Prism 7.02). Comparisons between the a CSF/serum albumin groups and levels of NFL was performed using one-way ANOVA with Bonferroni post hoc using SPSS 23.0 (SPSS, Chicago, IL, USA)

\section{Acknowledgements}

The authors thank the patients and their families for participating in this research. We are grateful for the skillful technical assistance of Rita Grandér.

This study was supported by grants from the Swedish Research Council, the Knut and Alice Wallenberg Foundation, Swedish State Support for Clinical Research, the Wolfson Foundation, Alzheimerfonden, the Swedish Society of Medicine, the Swedish Radiation Safety Authority, the Swedish Childhood Cancer Foundation (Barncancerfonden), the Jubilee Clinic Anticancer Research fund, the Edith Jacobsson Donation Fund, the Lions Cancer Foundation, the Frimurare Barnhus Foundation and the Cerebral Palsy Alliance (Australia).

\section{Conflict of interest:}

$\mathrm{KB}$ and $\mathrm{HZ}$ are co-founders of Brain Biomarker Solutions in Gothenburg AB, a GU Venture-based platform company at the University of Gothenburg. The other authors declare that they have no conflict of interest.

\section{References}

Anckarsater, R., Anckarsater, H., Bromander, S., Blennow, K., Wass, C., Zetterberg, H., 2014. Non-neurological surgery and cerebrospinal fluid biomarkers for neuronal and astroglial integrity. J Neural Transm (Vienna). 121, 649-53.

Bacioglu, M., Maia, L.F., Preische, O., Schelle, J., Apel, A., Kaeser, S.A., Schweighauser, M., Eninger, T., Lambert, M., Pilotto, A., Shimshek, D.R., Neumann, U., Kahle, P.J., 
Staufenbiel, M., Neumann, M., Maetzler, W., Kuhle, J., Jucker, M., 2016. Neurofilament Light Chain in Blood and CSF as Marker of Disease Progression in Mouse Models and in Neurodegenerative Diseases. Neuron. 91, 56-66.

Blomstrand, M., Kalm, M., Grander, R., Bjork-Eriksson, T., Blomgren, K., 2014. Different reactions to irradiation in the juvenile and adult hippocampus. Int J Radiat Biol. 90, 807-15.

Bolouri, H., Savman, K., Wang, W., Thomas, A., Maurer, N., Dullaghan, E., Fjell, C.D., Ek, C.J., Hagberg, H., Hancock, R.E., Brown, K.L., Mallard, C., 2014. Innate defense regulator peptide 1018 protects against perinatal brain injury. Ann Neurol. 75, 395-410.

Duffner, P.K., 2004. Long-term effects of radiation therapy on cognitive and endocrine function in children with leukemia and brain tumors. Neurologist. 10, 293-310.

Duffner, P.K., 2010. Risk factors for cognitive decline in children treated for brain tumors. Eur J Paediatr Neurol. 14, 106-15.

Ek, C.J., Habgood, M.D., Dziegielewska, K.M., Potter, A., Saunders, N.R., 2001. Permeability and route of entry for lipid-insoluble molecules across brain barriers in developing Monodelphis domestica. J Physiol. 536, 841-53.

Ek, C.J., D'Angelo, B., Baburamani, A.A., Lehner, C., Leverin, A.L., Smith, P.L., Nilsson, H., Svedin, P., Hagberg, H., Mallard, C., 2015. Brain barrier properties and cerebral blood flow in neonatal mice exposed to cerebral hypoxia-ischemia. J Cereb Blood Flow Metab. 35, 818-27.

Fouladi, M., Gilger, E., Kocak, M., Wallace, D., Buchanan, G., Reeves, C., Robbins, N., Merchant, T., Kun, L.E., Khan, R., Gajjar, A., Mulhern, R., 2005. Intellectual and functional outcome of children 3 years old or younger who have CNS malignancies. J Clin Oncol. 23, 7152-60.

Friede, R.L., Samorajski, T., 1970. Axon caliber related to neurofilaments and microtubules in sciatic nerve fibers of rats and mice. Anat Rec. 167, 379-87.

Gisslen, M., Price, R.W., Andreasson, U., Norgren, N., Nilsson, S., Hagberg, L., Fuchs, D., Spudich, S., Blennow, K., Zetterberg, H., 2016. Plasma Concentration of the Neurofilament Light Protein (NFL) is a Biomarker of CNS Injury in HIV Infection: A Cross-Sectional Study. EBioMedicine. 3, 135-40.

Gurney, J.G., Krull, K.R., Kadan-Lottick, N., Nicholson, H.S., Nathan, P.C., Zebrack, B., Tersak, J.M., Ness, K.K., 2009. Social outcomes in the Childhood Cancer Survivor Study cohort. J Clin Oncol. 27, 2390-5.

Han, J.W., Kwon, S.Y., Won, S.C., Shin, Y.J., Ko, J.H., Lyu, C.J., 2009. Comprehensive clinical follow-up of late effects in childhood cancer survivors shows the need for early and well-timed intervention. Ann Oncol.

Kalm, M., Fukuda, A., Fukuda, H., Ohrfelt, A., Lannering, B., Bjork-Eriksson, T., Blennow, K., Marky, I., Blomgren, K., 2009. Transient inflammation in neurogenic regions after irradiation of the developing brain. Radiat Res. 171, 66-76. 
Kalm, M., Abel, E., Wasling, P., Nyman, J., Hietala, M.A., Bremell, D., Hagberg, L., Elam, M., Blennow, K., Bjork-Eriksson, T., Zetterberg, H., 2014. Neurochemical evidence of potential neurotoxicity after prophylactic cranial irradiation. Int J Radiat Oncol Biol Phys. 89, 607-14.

Kuhle, J., Barro, C., Andreasson, U., Derfuss, T., Lindberg, R., Sandelius, A., Liman, V., Norgren, N., Blennow, K., Zetterberg, H., 2016a. Comparison of three analytical platforms for quantification of the neurofilament light chain in blood samples: ELISA, electrochemiluminescence immunoassay and Simoa. Clin Chem Lab Med. 54, 1655-61.

Kuhle, J., Barro, C., Disanto, G., Mathias, A., Soneson, C., Bonnier, G., Yaldizli, O., Regeniter, A., Derfuss, T., Canales, M., Schluep, M., Du Pasquier, R., Krueger, G., Granziera, C., 2016b. Serum neurofilament light chain in early relapsing remitting MS is increased and correlates with CSF levels and with MRI measures of disease severity. Mult Scler. 22, 1550-1559.

Lahteenmaki, P.M., Harila-Saari, A., Pukkala, E.I., Kyyronen, P., Salmi, T.T., Sankila, R., 2007. Scholastic achievements of children with brain tumors at the end of comprehensive education: a nationwide, register-based study. Neurology. 69, 296-305.

Lannering, B., Sandstrom, P.E., Holm, S., Lundgren, J., Pfeifer, S., Samuelsson, U., Stromberg, B., Gustafsson, G., 2009. Classification, incidence and survival analyses of children with CNS tumours diagnosed in Sweden 1984-2005. Acta paediatrica. 98, 1620-7.

Lee, M.K., Cleveland, D.W., 1996. Neuronal intermediate filaments. Annu Rev Neurosci. 19, 187-217.

Ljungqvist, J., Zetterberg, H., Mitsis, M., Blennow, K., Skoglund, T., 2016. Serum Neurofilament Light Protein as a Marker for Diffuse Axonal Injury: Results from a Case Series Study. J Neurotrauma.

Lyck, R., Enzmann, G., 2015. The physiological roles of ICAM-1 and ICAM-2 in neutrophil migration into tissues. Curr Opin Hematol. 22, 53-9.

Makale, M.T., McDonald, C.R., Hattangadi-Gluth, J.A., Kesari, S., 2017. Mechanisms of radiotherapy-associated cognitive disability in patients with brain tumours. Nat Rev Neurol. 13, 52-64.

Mattsson, N., Andreasson, U., Zetterberg, H., Blennow, K., 2017. Plasma neurofilament light as a biomarker for neurodegeneration in Alzheimer's disease. JAMA Neurol. . In press.

Mulhern, R.K., Merchant, T.E., Gajjar, A., Reddick, W.E., Kun, L.E., 2004. Late neurocognitive sequelae in survivors of brain tumours in childhood. Lancet Oncol. 5, 399-408.

Ostrom, Q.T., Gittleman, H., Liao, P., Rouse, C., Chen, Y., Dowling, J., Wolinsky, Y., Kruchko, C., Barnholtz-Sloan, J., 2014. CBTRUS statistical report: primary brain and central 
nervous system tumors diagnosed in the United States in 2007-2011. Neuro Oncol. 16 Suppl 4, iv1-63.

Petzold, A., 2005. Neurofilament phosphoforms: surrogate markers for axonal injury, degeneration and loss. J Neurol Sci. 233, 183-98.

Rohrer, J.D., Woollacott, I.O., Dick, K.M., Brotherhood, E., Gordon, E., Fellows, A., Toombs, J., Druyeh, R., Cardoso, M.J., Ourselin, S., Nicholas, J.M., Norgren, N., Mead, S., Andreasson, U., Blennow, K., Schott, J.M., Fox, N.C., Warren, J.D., Zetterberg, H., 2016. Serum neurofilament light chain protein is a measure of disease intensity in frontotemporal dementia. Neurology. 87, 1329-36.

Rojas, J.C., Karydas, A., Bang, J., Tsai, R.M., Blennow, K., Liman, V., Kramer, J.H., Rosen, H., Miller, B.L., Zetterberg, H., Boxer, A.L., 2016. Plasma neurofilament light chain predicts progression in progressive supranuclear palsy. Ann Clin Transl Neurol. 3, 216-25.

Roughton, K., Andreasson, U., Blomgren, K., Kalm, M., 2013. Lipopolysaccharide-Induced Inflammation Aggravates Irradiation-Induced Injury to the Young Mouse Brain. Dev Neurosci.

Sandor, N., Walter, F.R., Bocsik, A., Santha, P., Schilling-Toth, B., Lener, V., Varga, Z., Kahan, Z., Deli, M.A., Safrany, G., Hegyesi, H., 2014. Low dose cranial irradiation-induced cerebrovascular damage is reversible in mice. PLoS One. 9, e112397.

Semple, B.D., Blomgren, K., Gimlin, K., Ferriero, D.M., Noble-Haeusslein, L.J., 2013. Brain development in rodents and humans: Identifying benchmarks of maturation and vulnerability to injury across species. Prog Neurobiol.

Shahim, P., Gren, M., Liman, V., Andreasson, U., Norgren, N., Tegner, Y., Mattsson, N., Andreasen, N., Ost, M., Zetterberg, H., Nellgard, B., Blennow, K., 2016. Serum neurofilament light protein predicts clinical outcome in traumatic brain injury. Sci Rep. 6, 36791.

Tambuyzer, B.R., Ponsaerts, P., Nouwen, E.J., 2009. Microglia: gatekeepers of central nervous system immunology. J Leukoc Biol. 85, 352-70.

Tibbling, G., Link, H., Ohman, S., 1977. Principles of albumin and IgG analyses in neurological disorders. I. Establishment of reference values. Scand J Clin Lab Invest. 37, 385-90.

Ubogu, E.E., Cossoy, M.B., Ransohoff, R.M., 2006. The expression and function of chemokines involved in CNS inflammation. Trends Pharmacol Sci. 27, 48-55.

Weydt, P., Oeckl, P., Huss, A., Muller, K., Volk, A.E., Kuhle, J., Knehr, A., Andersen, P.M., Prudlo, J., Steinacker, P., Weishaupt, J.H., Ludolph, A.C., Otto, M., 2016. Neurofilament levels as biomarkers in asymptomatic and symptomatic familial amyotrophic lateral sclerosis. Ann Neurol. 79, 152-8.

Yao, Y., Tsirka, S.E., 2014. Monocyte chemoattractant protein-1 and the blood-brain barrier. Cell Mol Life Sci. 71, 683-97. 
Zarbock, A., Ley, K., McEver, R.P., Hidalgo, A., 2011. Leukocyte ligands for endothelial selectins: specialized glycoconjugates that mediate rolling and signaling under flow. Blood. 118, 6743-51. 